\title{
Téoros
}

Revue de recherche en tourisme

\section{Le tourisme autochtone}

Un produit d'avenir!

\section{Louise Séguin}

Volume 17, numéro 2, été 1998

L'industrie touristique autochtone

URI : https://id.erudit.org/iderudit/1072268ar

DOI : https://doi.org/10.7202/1072268ar

Aller au sommaire du numéro

Éditeur(s)

Université du Québec à Montréal

ISSN

0712-8657 (imprimé)

1923-2705 (numérique)

Découvrir la revue

\section{Citer cet article}

Séguin, L. (1998). Le tourisme autochtone : un produit d'avenir ! Téoros, 17(2),

13-16. https://doi.org/10.7202/1072268ar

Ce document est protégé par la loi sur le droit d'auteur. L'utilisation des services d’Érudit (y compris la reproduction) est assujettie à sa politique d'utilisation que vous pouvez consulter en ligne.

https://apropos.erudit.org/fr/usagers/politique-dutilisation/
Cet article est diffusé et préservé par Érudit.

Érudit est un consortium interuniversitaire sans but lucratif composé de l’Université de Montréal, l'Université Laval et l'Université du Québec à Montréal. Il a pour mission la promotion et la valorisation de la recherche. https://www.erudit.org/fr/ 


\section{tan...... \\ LE TOURISME AUTOCHTONE \\ UN PRODUIT D'AVENIR!}

\section{Louise Séguin}

Au Québec, développer le tourisme en milieu autochtone constitue un défi quotidien pour tous ceux qui s'y engagent. La diversité socioculturelle et économique des 54 commanautés autochtones y est certes pour quelque chose.

Chez nous, les Autochtones forment 11 nations distinctes réparties dans 13 de nos 19 régions touristiques, du sud au nord et d'est en ouest. La taille des localités varie considérablement. Ainsi, le village inuit d'Aupaluk, dans la baie d'Ungava, compte à peine 150 personnes alors que plus de 8000 Mohawks vivent à Kahnawake, près de Montréal.

\section{DE KAHNAWAKE À KUUJJUAQ}

Pour reprendre l'approche du guide touristique Le Quebec autochtone ${ }^{1}$, on peut situer les communautés dans cinq types d'environnement touristique. Il y a d'abord les communautés * urbaines *, dont font partie notamment Kahnawake et Wendake. Ces dernières sont situées à quelques minutes des centres-villes de Montréal et de Québec, pôles majeurs de fréquentation touristique au Québec. Les communautés dites * rurales *, sans être aussi près des centres urbains, en sont relativement peu éloignées. Elles ont souvent l'allure de villages ruraux, comme c'est le cas pour Odanak près de Nicolet, Mashteuiatsh près de Roberval, Kitigan Zibi à côté de Maniwaki ou Pikogan à quelques minutes d'Amos.

Plusieurs autres communautés sont dites « côtières \%. C'est là que vivent par exemple les Micmacs de la Baie-des-Chaleurs (Listuguj et Gesgapegiag) et les Montagnais de la Côte-Nord (Betsiamites, Mingan, Natashquan). Ces villages sont relativement isolés, mais tout de même reliés au réseau routier. Les communautés * forestières * aussi sont accessibles par la route, mais elles sont très isolées, autant géographiquement que culturellement. Les Atikamekw de la Haute-Mauricie (Obedjiwan, Weymontachie et Manawan), les Cris du Moyen-Nord (Ouje-Bougoumou, Mistissini et Waswanipi par exemple), ainsi que les Algonquins de plusieurs villages de l'Abitibi-Témiscamingue en font partie.

Enfin, tout à fait au nord, dans la * nouvelle $*$ région touristique Nord-duQuébec, se trouvent les communautés « côtières nordiques $w$ : les communautés cries de la Baie-James ainsi que les 11 communautés inuit, qui parsèment les 1 600 kilomètres de littoral de la baie et du détroit d'Hudson, ainsi que de la baie d'Ungava. Ces dernières ne sont accessibles que par avion.

Les environnements physique et géographique ont une importance capitale sur l'organisation du tourisme autochtone, tant en ce qui concerne les forces et les faiblesses des produits offerts (ou en développement) qu' en ce qui concerne leur mise en marché.

\section{UN ENGOUEMENT RÉCENT}

Il y a quelques décennies à peine, la majorité des Autochtones du Québec vivaient encore selon un mode de vie plus ou moins nomade. La sédentarisation rapide et la vie en village sont des phénomènes très récents qui ont demandé des efforts considérables aux populations amérindiennes et inuit, notamment celles qui sont éloignées des centres urbains. Les communautés ont été longtemps peu ouvertes aux visiteurs, trop occupées à organiser leurs services de base.

Cependant, le tourisme est graduellement devenu une réalité économique à bien des endroits. Un nombre croissant de communautés et d'entrepreneurs privés s'y intéressent, plusieurs ont même commencé à le développer sérieusement. Alors qu'il y a 15 ans à peine les seuls touristes dans les communautés étaient des travailleurs de l'extérieur, aujourd' hui, on y aperçoit fréquemment des centaines de visiteurs étrangers. La richesse des cultures autochtones exerce un attrait considérable, particulièrement auprès des clientèles européennes.

En fait, dans ce domaine particulier du tourisme, la demande est en quelque sorte arrivée avant l'offre. Dans bien des cas, les communautés ont vu et voient encore arriver des autobus de touristes sans posséder les infrastructures suffisantes pour les accueillir et, surtout, pour les retenir chez eux et leur offrir l'expérience touristique qu'ils recherchent.

Qu'est-ce que les Autochtones offrent comme produit, au juste ? Dans quels secteurs d'activités sont-ils les plus 


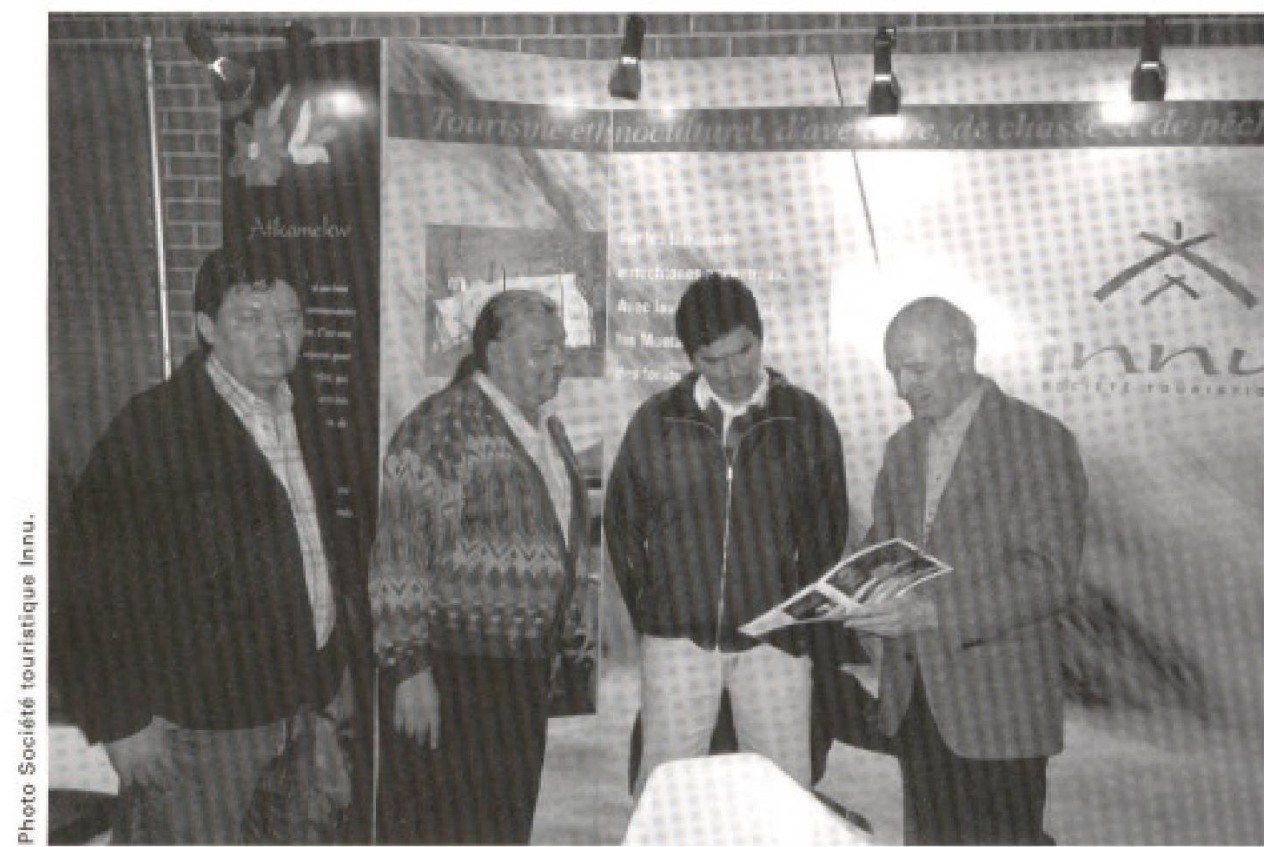

La diffusion d'information lors des Salons du tourisme.

actifs, les plus performants, les mieux connus ? Comment l'ensemble du produit s'insère-t-il au sein de l'industrie touristique régionale et québécoise ? Quelles sont les forces et les faiblesses des entreprises et de l'ensemble du produit touristique autochtone? Autant de questions qui intéressent Tourisme Québec et, de plus en plus, l'ensemble de l'industrie touristique québécoise.

C'est pourquoi, dans sa toute récente politique de développement touristique, Pour donner au monde le goat du Quebec, Tourisme Québec identifie le tourisme autochtone comme un produit en ếmergence et reconnaît l'importance de miser sur son développement. D'où l'intérêt à mieux connaître le produit et le contexte dans lequel il évolue.

\section{LES PRODUITS TOURISTIQUES AUTOCHTONES}

Fait peu surprenant puisqu'il fait appel à la connaissance intime que les Autochtones ont du territoire qu'ils fréquentent, le produit le mieux organisé et le plus vendu est, actuellement, le produit chasse et pêche. Au total, une cinquantaine des 700 pourvoiries du Québec appartiennent à des intếrêts autochtones de diverses nations, particulièrement les Inuit, les Montagnais, les Cris, les Algonquins, les Atikamekw, les Abénaquis et les Naskapis. La com- munauté montagnaise d'Essipit, par exemple, offre ce produit depuis plus de 15 ans.

Un certaine nombre de pourvoiries sont exploitées en partenariat avec des non Autochtones, notamment chez les Inuit. Chez les pourvoyeurs du Nord, l'activité principale est la chasse au caribou, suivie de la pêche. La région Nord-duQuébec recèle un potentiel important grâce aux imposants troupeaux de caribous qui sillonnent le territoire. A elles seules, les pourvoiries inuit reçoivent de 2500 à 3000 clients par année. Ceux-ci viennent majoritairement des États-Unis et déboursent, pour un séjour d'une semaine, entre 3000 et 5000 dollars américains, ce qui amène des retombées économiques importantes dans une région qui en a bien besoin. La commercialisation des pourvoiries inuit se fait d'ailleurs principalement sur le marché américain.

Compte tenu de l'évolution du marché de la pourvoirie et des exigences toujours plus grandes de la clientèle, les installations en place devront éventuellement être améliorées, d'une part, pour rehausser la qualité du produit et, d'autre part, pour diversifier les activités offertes.

Par ailleurs, bien que ne bénéficiant pas d'une mise en marché ni d'une structure très organisée, les visites des villages constituent le deuxième produit touristique autochtone d'importance. Une trentaine de communautés offrent cette activité où le volet interprétation culturelle est particulièrement recherché. Dans quelques communautés, les visites sont libres, alors que dans d'autres, elles sont organisées et encadrées.

C'est le cas à Ouje-Bougoumou. Le personnel de Tourisme Ouje-Bougoumou accueille le visiteur et lui offre une tournée complète du village construit il y a quelques années à peine et conçu par l'architecte Douglas Cardinal. La tournée s"accompagne de commentaires sur l'histoire tout à fait particulière du village et de la communauté crie qui l' habite. Le visiteur a l'occasion de se rendre à un parc adjacent où sont reproduites les diverses habitations que les Cris ont utilisées à travers les âges, en plus de s'initier aux mets traditionnels et à la culture crie.

Les communautés situées près de routes achalandées (ex. : Odanak, Mashteuiatsh. Wendake, Ouje-Bougoumou, Kahnawake, etc.) sont celles qui reçoivent le plus grand nombre de visiteurs. On offre géneralement à ces derniers la visite d'un musée et des produits d'artisanat, des attractions comme des villages historiques reconstitués, ainsi que des manifestations touristiques telles que pow-wow et activités d'animation culturelle. Cette année, la communauté de Kahnawake innove en offrant un forfait qui combine souper et thêtre, où l'on attend quelques milliers de visiteurs au cours de la saison estivale.

Un tel type de tourisme vise les voyageurs qui effectuent des circuits routiers en automobile ou en autobus et génère un volume important de visiteurs. Il n'existe pas de statistiques officielles à ce sujet. On peut toutefois présumer qu'une communauté comme Wendake, qui offre diverses activités telles que des visites guidées du vieux village et du musée Aroüiane, des boutiques d'artisanat, l'animation du site huron reconstitué Onhoüa Chetek8e, profite de sa situation géographique privilégiée dans la région immédiate de Québec et reçoit sans doute plusieurs dizaines de milliers de visiteurs annuellement.

Compte tenu du nombre important de communautés autochtones et de leur répartition dans l'ensemble des régions du Québec, ce produit offre un potentiel intéressant de développement. Dans plusieurs communautés, il est toutefois opportun de développer des attractions et des 
activités complémentaires qui contribueront à retenir les touristes et à leur offrir davantage qu"un simple arrêt dans une boutique d'artisanat.

L'écotourisme, l'aventure et le plein air sont des activités de plus en plus populaires au Québec. Les territoires habités par plusieurs nations autochtones sont particulièrement propices au développement de tels produits et il y a de nombreux projets en croissance ou en émergence. Certaines communautés cries de la BaieJames et algonquines d'Abitibi-Témiscamingue proposent, sur demande, des excursions de canot-camping et des randonnées d'observation de la faune. Essipit, sur la Cote-Nord, offre depuis quelques années des croisiềres d'observation des baleines fort appréciées.

L'expérience ethnoculturelle, qui prend généralement la forme de séjours en forêt et d'activités de plein air axées sur l'interprétation de la culture autochtone, est également un secteur en développement. A titre d'exemple, l'entreprise montagnaise Aventure Mikuan II offre depuis plusieurs années des excursions et des séjours en forêt. C'est l'un des produits commercialises par Tours innu, grossiste réceptif autochtone spécialisé en tourisme ethnoculturel et aventure douce chez les Amérindiens. Nuuhchimi Wiinuu, une petite entreprise familiale d'OujeBougoumou, propose également le même type d'activités, enrichies de toutes les connaissances des promoteurs sur la culture, 1'histoire et les traditions cries.

Toutefois, de façon générale, on constate que le tourisme d'aventure, l'écotourisme et le tourisme ethnoculturel en milieu autochtone demeurent encore peu développés. La moitié des produits offerts sont en démarrage, peu commercialisés et ne reçoivent qu' une clientèle limitée.

\section{LES OBSTACLES AU DÉVELOPPEMENT}

Il n'est pas facile de démarrer une entreprise dans le secteur touristique. Plusieurs des quelque 29000 petites entreprises touristiques au Québec peuvent en témoigner. Dans bien des régions, les activités sont saisonnieres. L'acces aux sources de financement est ardu, car les institutions financières considèrent le secteur touristique comme un secteur à risque. Enfin, il n'est pas suffisant d'avoir un bon pro- duit, encore faut-il qu'il soit connu, et les activités de promotion et de commercialisation sont en général fort coûteuses. Il en résulte que les entreprises réussissent difficilement à dépasser le stade artisanal.

Dans le cas des entreprises autochtones, les problèmes sont accentués par de multiples contraintes découlant des contextes légal, socioéconomique, culturel et géographique qui influencent le développement des communautés. Dans un document sur les orientations du gouvernement du Québec en matière autochtone ${ }^{2}$, rendu public récemment, le Secrétariat aux affaires autochtones identifie les difficultés qui font obstacle au développement économique. Parmi celles-ci, plusieurs concernent le secteur touristique : manque de formation de la main-d'cuvre ; faible organisation, eloignement et isolement des entreprises et du milieu autochtone en général par rapport aux réseaux. et aux marchés ; attitudes à l'endroit du monde des affaires et opposition entre le développement moderne et les valeurs traditionnelles ; valorisation moindre de l'entreprise privée ; méconnaissance entre les Autochtones et les non Autochtones ; inadéquation des mesures d'interventions gouvernementales et problèmes d'accès aux capitaux ${ }^{3}$.

De façon générale, les statistiques démontrent qu'en milieu autochtone le niveau de vie et la scolarité sont plus faibles que dans l'ensemble du Québec et que le taux

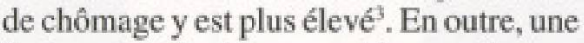
forte poussée démographique crée une pression importante dans le milieu.

De tels éléments exercent une influence certaine sur le développement du tourisme. A l'échelle des communautés et à celle des nations, on ressent un urgent besoin de créer des emplois. Pour plusieurs, le développement touristique apparaît comme un secteur où l'histoire et les connaissances ancestrales du territoire, de sa faune et de sa flore peuvent štre mises en valeur. Les attentes à $1^{\dagger}$ égard des retombées économiques provenant du tourisme sont donc élevées.

Le financement des projets demeure l'obstacle le plus important auquel les promoteurs autochtones en tourisme doivent faire face. Peu d'entre eux disposent de capitaux d'investissement suffisants. Les terres situées dans les réserves sont des terres fédérales dont les Amérindiens ne sont pas propriétaires et, de surcroît, ceux-ci ne peuvent proposer leurs biens en garantie de leurs prêts. En effet, sur les réserves, en vertu de la Loi sur les Indiens, les biens des Amérindiens sont déclarés insaisissables. Les institutions financières se montrent donc extrêmement réticentes à financer des projets dans de telles conditions. Elles exigent des garanties et un investissement très élevés de la part des promoteurs, ce qui ajoute aux difficultés de démarrage et de rentabilisation de l'entreprise. Cette conjoncture rend donc les promoteurs autochtones très dépendants des programmes d'aide gouvernementaux.

Par ailleurs, les populations sont peu nombreuses et le bassin de main-d'cuvre locale est restreint. L'accès à la formation spécialisée se révèle difficile, tant pour le personnel que pour les gestionnaires d'entreprises, notamment en raison de la faible scolarisation et de l'éloignement des grands centres. De plus, les entrepreneurs sont souvent isoles, ont peu de contacts avec les réseaux touristiques et, ainsi, peu d'occasions de développer des alliances et des partenariats.

\section{UN PRODUIT ATTRAYANT...}

Examinons maintenant les forces et les faiblesses du produit. D'une part, l'image : tout le monde s'entend pour reconnaittre que les Autochtones jouissent sur les marchés internationaux, surtout en Europe, d'une image spécifique, très claire, fortement positive. Cette image constitue une force majeure du produit.

De plus, la majorité des communautés autochtones sont localisées dans la nature, au cour de réseaux de lacs et de rivières. Cela leur ouvre la porte au développement de produits d'aventure, de plein air et d'écotourisme basés sur leur culture propre. Ces particularités permettent, bien sûr, la tenue d'activités d'interprétation ethnoculturelle.

Par ailleurs, quelques sociétés et agences autochtones de développement et de commercialisation du produit sont en émergence. Ainsi, la Société touristique Innu, mise sur pied au début des années 1990 , représente les entreprises et les communautés atikamekw et montagnaise. Elle a créé une agence réceptive, Tours innu, qui commercialise les produits autochtones, tel que mentionné précédemment. 
Du côté du Nord-du-Québec, les Inuit du Nunavik ont, pour leur part, mis sur pied leur propre association touristique et les Cris sont également en voie de le faire. Ces deux groupes s'associeront à Tourisme Baie-James, l'organisme qui représente les localités non autochtones de la BaieJames, pour former l'Association touristique régionale Nord-du-Québec, la dixneuvième association touristique régionale reconnue par Tourisme Québec.

On peut qualifier ces regroupements de * forces vives du milieu $\%$. Ils sont des outils importants, dotés d'une connaissance spécialisée à la fois de la situation des Autochtones et des exigences du marché de l'industrie touristique. Le développement harmonieux du tourisme autochtone ne saurait certainement se faire sans une prise en charge par le milieu luimểme, et ces groupes sont tout désignés pour devenir des interlocuteurs essentiels auprès des instances gouvernementales et des partenaires des régions.

\section{...MAIS PEU STRUCTURÉ}

Cependant, le produit autochtone est actuellement peu intégré à l'ensemble du produit touristique québécois. Sauf quelques rares exceptions, il n'y a à peu près pas d'interactions entre les communautés ou les entreprises autochtones et les associations touristiques régionales. $\AA$ l'instar des communautés, le produit autochtone est peu visible et peu connu localement. Les promoteurs ne peuvent donc pas bénéficier des retombées des campagnes de marketing et de promotion mises sur pied par les régions. Pourtant, un produit autochtone bien développé pourrait représenter un complément fort convoité dans les régions où il est présent. Pourquoi pas, en effet, un circuit montagnais sur la Côte-Nord, micmac en Gaspésie ou algonquin en Abitibi-Témiscamingue, qui intégrerait produits et infrastructures autochtones et non autochtones?

La confrontation entre les valeurs traditionnelles des sociétés autochtones, axées sur le développement communautaire, et les exigences commerciales, reliées au développement touristique, agit également comme frein au développement. Étant donné l'importance de l'engagement de tous les individus de la communauté dans une démarche d'accueil, les promoteurs doivent compter sur l'appui de la population locale. Celle-ci craint parfois, avec ou sans raison, de se voir envahir par des hordes de touristes.

Par ailleurs, l'industrie touristique autochtone ne dispose pas encore de normes de qualité des produits et services. Il n'existe pas non plus de tradition de garantie de livraison du produit tel que commercialisé. Dans certains cas, la demande est arrivée avant que l'offre ne soit complètement prête et la mise en place accélérée d'activités a entraîné des situations où le produit n'a pas été livré tel qu'annoneé. Cette situation n'a pas aidé à la vente du produit dans les réseaux de distribution, rendant certains voyagistes craintifs ou méfiants à l'égard de tout ce qui est autochtone. Là encore, les regroupements d'entreprises doivent jouer un rôle en rendant compte auprès des voyagistes de l'évolution du produit.

La culture constitue l'essence et la force d'attraction du produit touristique autochtone. Cependant, dans bien des cas, les guides et les animateurs autochtones disposent de peu d'outils pour interpréter I'histoire et la culture locales. Il serait opportun, à ce stade-ci, que les organismes autochtones de développement économique et culturel s'intéressent également à cet aspect et contribuent à doter les promoteurs d'outils adaptés à leurs besoins et aux attentes des touristes.

\section{TOUT COMPTE FAIT...}

Malgré les limites, tout ne reste pas à faire en ce qui concerne le développement touristique chez les Autochtones... La situation a évolué énormément depuis dix ou quinze ans et déjà des pas importants ont été faits, des étapes franchies. Nombreux sont ceux qui ont consolidé leur expérience du domaine touristique, que ce soit dans le développement de leur entreprise, dans l'établissement de réseaux d'affaires ou dans la commercialisation de leurs produits.

Dans diverses régions du Québec, de plus en plus de représentants autochtones sont invités à joindre les rangs des associations et des conseils de développement économique et touristique et diverses formes de partenariat entre entrepreneurs autochtones et non autochtones voient le jour.
On assiste présentement à une volonté des Autochtones d'élargir leurs réseaux à l'échelle de l'ensemble des nations du Québec et à l'ensemble de l'industrie touristique québécoise. Signe des temps et de l'intérêt suscité par le tourisme autochtone, le ministre délégué au Tourisme, David Cliche, a invité Aurélien Gill, président de la Société touristique Innu et homme d'affaires montagnais, à siéger au Forum permanent de l'industrie touristique.

Tout compte fait, le tourisme en milieu autochtone est porteur d'un avenir prometteur. Des défis stimulants attendent ses acteurs : consolider ou améliorer la qualité du produit offert, diversifier les activités, consolider les réseaux d'échange et les partenariats, structurer et intensifier les efforts de promotion et de commercialisation et développer de nouveaux marchés.

L'auteure est coordonnatrice aux affaires et déléguée touristique du Nord-du-Québec et de l'Abitibi-Témiscamingue à Tourisme Québec. Originaire de Rouyn-Noranda, elle a été journaliste à la revue Rencontre du Secrétariat aux affaires autochtones et, auparavant, responsable administrative de la Cour itinérante qui dessert les communautes crie et inuit. Louise Séguin a habité le Nunavik et le Nunavut pendant plusieurs annés.

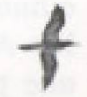

\section{NOTES}

1 Les éditions La griffe de l'aigle (1996), Le Québec autochtone, Wendake, 288 p.

2 Secrétariat aux affaires autochtones (1998), Partenariat, développement, action, Orientations du gouvernement du Québec, Québec, $40 \mathrm{p}$.

3 Secrétariat aux affaires autochtones (1998).

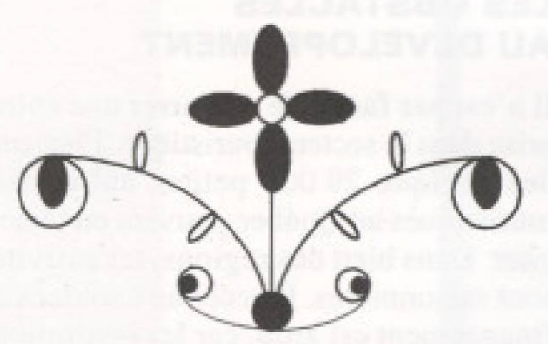

